\title{
Fuzzy based Load and Energy Aware Multipath Routing for Mobile Ad Hoc Networks
}

\author{
M. Ali \\ Caledonian College of \\ Engineering, Oman
}

\author{
B. G. Stewart \\ Glasgow Caledonian University, \\ Scotland, UK
}

A. Vallavaraj

Caledonian College of Engineering, Oman

\author{
A. Shahrabi \\ Glasgow Caledonian University, \\ Scotland, UK
}

\begin{abstract}
Routing is a challenging task in Mobile Ad hoc Networks (MANET) due to their dynamic topology and lack of central administration. As a consequence of un-predictable topology changes of such networks, routing protocols employed need to accurately capture the delay, load, available bandwidth and residual node energy at various locations of the network for effective energy and load balancing. This paper presents a fuzzy logic based scheme that ensures delay, load and energy aware routing to avoid congestion and minimise end-to-end delay in MANETs. In the proposed approach, forwarding delay, average load, available bandwidth and residual battery energy at a mobile node are given as inputs to a fuzzy inference engine to determine the traffic distribution possibility from that node based on the given fuzzy rules. Based on the output from the fuzzy system, traffic is distributed over fail-safe multiple routes to reduce the load at a congested node. Through simulation results, we show that our approach reduces end-to-end delay, packet drop and average energy consumption and increases packet delivery ratio for constant bit rate (CBR) traffic when compared with the popular Ad hoc On-demand Multipath Distance Vector (AOMDV) routing protocol.
\end{abstract}

\section{Key Words}

MANET, Fuzzy Logic, Load Balancing, Multipath Routing.

\section{INTRODUCTION}

MANETs are infrastructure-less multi-hop wireless networks, formed by a group of mobile nodes which cooperatively maintain network connectivity. In the absence of dedicated routers as in fixed networks, a mobile node in a MANET needs to act as a node as well as a router. Since there is no fixed infrastructure, MANETs are rapidly deployable in scenarios such as disaster recovery, business meetings, collaborations, communication over rugged terrains etc., where establishing infrastructure networks is either impractical or not cost effective. In such networks mobile nodes normally communicate via wireless channels which are bandwidthconstrained, error-prone, and insecure and hence they have significantly lower data transmission capability compared to traditional fixed networks. Moreover, since nodes in such networks are normally portable hand-held devices, the limited power of energy sources carried by them limits the applications and services that can be provided by such networks. Because of mobility and limited energy of nodes, topologies of MANETs are highly dynamic, where nodes may join the network, change their position or disappear at any time. Constraints such as high node mobility, bandwidth-constrained wireless links, limited battery power, contention for the shared wireless medium and the highly dynamic nature of a MANET that results in frequent and unpredictable changes of network topology make routing in MANETs a difficult and complex task [1]. Routing protocols need to cope with such situations, and maintain continued communication between nodes in the presence of such abrupt changes in the network due to node failures or mobility. Such constraints demand delay, congestion and energy aware adaptive routing that maximises node life time and evenly distributes traffic in the network.

\subsection{Routing in MANETs}

In traditional infrastructure networks, routing protocols run in specialised nodes which are optimised for that purpose with plenty of resources such as energy, memory, processing power etc. On the other hand, routing protocols in MANETs should run on normal resource-constrained nodes which form a topology that is highly dynamic and unpredictable. In MANETs, though mobile nodes that are in range can directly communicate with each other, data meant for other nonneighbouring nodes is transmitted using a series of intermediate nodes which act as routers. MANETs suffer from traditional inherited problems of wireless communication such as lack of absolute boundaries of the wireless medium, interference from other signals, less reliability of the wireless medium, time-varying and asymmetric properties of the channel, hidden and exposed node problems etc. However, they also have problems due to the nature and characteristics that are specific to MANETs such as: multi-hop routing, resource constrained nodes, autonomous operation, unpredictable environment, dynamically changing topology, network scalability etc.

Routing schemes normally employed in MANETs can be broadly classified into proactive or table-driven and reactive or on-demand categories. Proactive schemes require each node to maintain a table of routes to every other node in the network. The drawback is that to keep routing tables refreshed, routing information messages require to be periodically exchanged between nodes. However, on-demand protocols compute routes based on demand, as and when required, which make them more scalable to large dynamic networks $[1,2]$.

\subsection{Multipath Routing in MANETs}

The majority of current MANET routing protocols use shortest single path routing with minimum hop count [1]. However studies have shown that single path routing based on minimum hop count metrics may not always give good results, mainly in MANETs that have nodes along the route that are congested or have reduced resources such as lower bandwidth or residual energy $[1,3]$. The identified routes in single path routing may also not be reliable because node mobility or link conditions could lead to frequent route breaks resulting in transmission failures leading to delay and congestion thus making routing unreliable. Moreover, the time taken by single path routing to recover from route failures may not be acceptable to many delay-sensitive applications [4]. The performance of single path on-demand routing protocols degrades sharply with increase in the number of nodes. In addition to this, the shortest 
hop metric overburdens nodes which are part of the shortest paths that very often lie in the center of the network [5]. Such overloaded nodes start getting depleted of energy quickly, become congested and start dropping packets sometimes leading to network partitions. Thus applying load balancing schemes to routing protocols becomes necessary to push traffic from the center of the network to less congested links for maximizing the network utilization [5].

To overcome the above limitations, multipath routing is suggested. In multipath routing, either all the discovered paths could be used simultaneously or one path used at any given time. In the former case, the traffic is distributed simultaneously using all the paths from source to destination. The fraction of traffic distributed in each path may be based on some cost metric such as aggregate bandwidth or average residual energy of the path. In the latter case, the protocol can select one from the many discovered routes as the main path based on some metric and the remaining routes used as alternative paths, to be selected when the main path fails. Load balancing is essential in MANETs which use multipath routing, where the load and congestion across the network need to be precisely captured and distributed to avoid bottlenecks and congestion $[4,6]$. Multipath routing in MANETs leads to many advantages including effective load balancing, higher aggregate bandwidth, route resilience, network scalability and faulttolerance. Spreading of traffic along multiple routes ensures load balancing, and alleviates congestion and delay in the network. Indeed higher aggregate bandwidth of a connection leads to higher throughput and less delay for the application. Further, due to the availability of simultaneous multiple paths between source and destination, multipath routing can provide route resilience provided that at least one of the paths exists. In multipath routing, however, achievable throughput of a flow may be limited due to radio interference between transmitting nodes in different paths that may be operating on the same channel. However, studies have shown that in dense MANETs, multipath routing provides enhanced throughput over singlepath routing [1,3]. To this end improved routing solutions which encapsulate and integrate many of these required features are preferred.

As a solution to the issues discussed above, this paper presents a fuzzy based load and energy aware multipath routing (FLEAMR) protocol for MANETs that is congestion and energy aware and also ensures load balancing and avoids congestion. The fuzzy logic based load distribution mechanism distributes load based on the forwarding delay, average load, available bandwidth and residual battery energy of a node over fail-safe multiple paths. The method for discovering fail-safe multiple paths is based on 'Scalable Multipath On-demand Routing (SMORT)' [7], that finds multiple fail-safe paths between source and destination nodes. A cross layer approach is followed by which the forwarding delay, available bandwidth, load and residual energy from lower layers are used for taking appropriate routing decisions.

The organisation of the paper is as follows: Section 2 presents related work in energy awareness, load balancing and congestion control in MANETs; Section 3 explains the parametric routing metrics used in the proposed model; Section 4 outlines the fuzzy based load distribution algorithm; Section 5 presents simulations and the analysis of some results, and finally conclusions are presented in Section 6.

\section{RELATED WORK}

Several publications have approached load balancing and energy awareness problems in MANETs [7-13]. Reddy and
Raghavan [7] proposed the 'scalable multipath on-demand routing' (SMORT) protocol, which uses the concept of secondary paths. The idea of SMORT is to provide multiple fail-safe secondary routes to the destination node from all the intermediate nodes on the main path. High scalability is the main feature of the protocol, making it suitable for highly mobile networks with high traffic loads. The protocol, however, is not energy aware.

Pham and Perreau [14] have analytically shown that in MANETs, shortest single path protocols have a tendency to use nodes located in the network center for a large number of routes. Thus nodes lying in the network centre are more engaged in shortest paths compared to non-centralised nodes, which may lead to bottle-necks and congestion. Based on this work, Souihli et al. [5] proposed a routing protocol by introducing a special metric called the 'degree of centrality' of a node. Their algorithm ensures load-balancing by using routes which are far from the geometric center of the network. This algorithm enhances network performance by means of improved load distribution in the network. However, the protocol being single-path has inherent limitations in that the selected path, though far from network center, may not satisfy the QoS and bandwidth requirements of the application. Further, the protocol is also not energy aware.

Many studies have shown that fuzzy logic can be successfully applied in network routing protocols [15]. A number of studies have proposed the application of fuzzy logic for performance improvements in MANET routing protocols [16-19]. Natsheh et al. [16] proposed an adaptive route lifetime approach using fuzzy logic based on the observation that the route lifetime value is one of the most important parameters to be considered while designing on-demand MANET routing protocols. This parameter determines how long the route stays active in the routing table and hence can be used for routing packets. As long as the route lifetime does not expire, the protocol neither attempts to discover a new route nor delete an existing route. The authors designed a new method called fuzzy active route timeout (ART) by defining fuzzy sets (membership functions) and an associated set of rules (rule base). They evaluated the proposed fuzzy ART in the ad hoc on demand distance vector (AODV) protocol which otherwise used a static ART. The adaptive fuzzy based ART resulted in improved performance with respect to packet delivery ratio, routing overhead and average end-to-end delay [16]. However their approach is not energy aware and is single path and hence may incur high route re-discovery delay in the event of route breaks due to node/link failure or mobility.

Ali and Fahad [17] proposed a fuzzy based energy aware routing protocol for MANETs where fuzzy based route selection is performed based on number of hops, packet queue occupancy and remaining energy along the paths. The authors extended the AODV protocol to take the remaining battery power, packet queue occupancy and number of intermediate hops as inputs to the fuzzy controller to produce the route costs to be used in the route selection process. The proposed fuzzy energy based routing method is evaluated and compared with conventional AODV routing in terms of packet delivery ratio, average end to end delay, and average energy consumption per node. Simulation results show that the proposed method improves the functionality and performance of the AODV routing protocol. Again, since the approach is based on single path routing, it may incur high route re-discovery delay in the event of route breaks due to node/link failure or mobility.

Oliveira and Braun [18] proposed a delay-based approach using fuzzy logic to improve Transmission Control Protocol 
(TCP) error detection in ad hoc networks. Their approach is based on the observation that regular TCP was not designed for highly dynamic networks such as MANETs, rather it was designed to work in wired networks where packet loss is due to network congestion. However in MANETs, such losses may occur not only by congestion but also due to high bit-error-rate of the wireless channels and due to mobility induced link interruptions. These types of losses induce TCP to mistakenly reduce its transmission rate seriously impairing data throughput. This problem can be solved by providing the TCP error detection mechanism with the actual cause of every packet loss. The authors make use of fuzzy logic theory for distinguishing between bit error and congestion induced losses, using round trip time (RTT) values as input variables. Simulation results have shown that the fuzzy engine may distinguish congestion from channel error conditions, and consequently assist the TCP error detection mechanism [22].

Misra et al. [19] proposed a fuzzy logic based energy efficient packet loss preventive routing protocol (FEEPRP) for wireless sensor networks which exploits the fuzzy decision making model for realising an energy-efficient secure routing protocol. FEEPRP ensures that reduced packet loss occurs due to various reasons such as the presence of malicious nodes and congestion by adopting a fuzzy logic-based approach to select energy efficient routes to a destination, thus preventing data loss and imparting security at the same time. Fuzzy logic is used to avoid the need for a complex mathematical model. The protocol chooses the metrics 'residual energy', 'hop count' and 'packets dropped', which monitor the current status of the network and results in selection of appropriate routes. Since these metrics are derived directly from the current status of the nodes, the route selected depends on the chosen metrics. The protocol is compared with DSR and AODV using Throughput, Packet loss and Energy efficiency as performance parameters and it is shown that the protocol improves these factors compared to the other two protocols. However, the protocol is not very scalable and cannot be applied in a MANET environment since additional delay may occur for route rediscovery in the event of route breaks due to node movements or failures.

All of the above developments have attempted to address the problems of energy awareness, load balancing, QoS provisioning and congestion control individually. The proposed FLEAMR protocol in this paper attempts to address all these issues in a single protocol which ensures delay and energy awareness, load balancing, congestion control and thereby improved QoS in MANETs. The proposed protocol gives all the advantages of multipath routing and also distributes traffic based on delay, load, bandwidth and residual energy level of nodes, which has not been attempted before in a single protocol for MANETs. FLEAMR adopts a cross layer approach and integrates a number of methods to provide performance improvements, which were otherwise achieved using multiple independent protocols.

\section{ROUTING METRICS}

This section introduces the various routing metrics employed in the FLEAMR protocol.

\subsection{Estimation of Node Delay}

The method proposed in [20] is used for estimation of forwarding delay at a node. The virtual carrier sensing mechanism of the IEEE 802.11 WLAN uses RTS-CTS frame exchange followed by an acknowledgement (ACK) for each data transmission to enhance data reliability. Two inter-frame spaces (DIFS - DCF Inter-Frame Space and SIFS - Short Inter-
Frame Space) are used for data transmission in normal mode. A mobile node that attempts to send packets undergoes transition through various states such as Idle, Packet_Arrival, Back-off and Attempt [20]. The forwarding delay $D_{\text {delay }}^{\bar{i}_{\text {art }} \text { at a }}$ mobile node $i$, which includes MAC contention and transmission delays, is calculated using equation (1) (as given in [3]):

$$
\begin{aligned}
D^{i} \text { delay }= & P_{\text {idle }}^{i}(D I F S) \times\left(D I F S+a v g \_b t+D A(i)+\right. \\
& \left(1-P^{i}{ }_{\text {idle }}(D I F S)\right) \times(S I F S+D B(i))+(L / R)
\end{aligned}
$$

where $P_{\text {idle }}^{i}(t)$ is the probability that node $i$ succeeds in detecting the channel is idle for time interval $t$ and is given by:

$$
P^{i} \text { idle }(t)=e^{-\lambda t},
$$

where $\lambda$ is the average packet arrival rate (including neighbour nodes) at mobile node $i$; $D A(i)$ is the expected delay encountered in the transmission attempt state of the node and is given by:

$$
\begin{aligned}
D A(i)= & P^{i}{ }_{\text {idle }}(\text { slot }) \times(R T S+2 * \text { SIFS }+ \text { CTS })+ \\
& \left(1-P^{i}{ }_{\text {idle }}(\text { slot })\right) \times(R T S+2 * \operatorname{SIFS}+D B(i))
\end{aligned}
$$

$D B(i)$ is the expected delay encountered during back-off and is given by:

$$
\begin{aligned}
D B(i)= & {\left[1 /\left\{P_{\text {idle }}^{i}(D I F S) \times P_{\text {idle }}^{i}(\text { slot })\right] \times\right.} \\
& {\left[P_{\text {idle }}^{i}(\text { DIFS }) \times\left(\text { DIFS }+ \text { avg } \_b t+R T S+2 * S I F S+\right.\right.} \\
& \left.\left.P^{i}{ }_{\text {idle }}(\text { slot }) \times C T S\right)\right]+\left[\left(1-P^{i} \text { idle }(\text { DIFS })\right) \times X\right]
\end{aligned}
$$

and where $a v g \_b t$ is the average back-off time of transmission evaluated through:

$$
\begin{aligned}
a v g_{-} b t & =\sum_{n=0}^{4}\left(P^{i}{ }_{\text {idle }}(\text { slot }) \times\left(1-P^{i}{ }^{i} \text { idle }(\text { slot })\right)^{n} \times 2^{n-1} \times W\right) \\
& +\left(1-P^{i} \text { idle }(\text { slot })\right)^{5} \times 2^{4} \times W .
\end{aligned}
$$

$L$ and $R$ are packet length and data rate respectively and $W$ is the contention window size; the term $\mathrm{X}$ is determined through:

$X=R T S+3 * S I F S+C T S+L+A C K$.

Since propagation delays of wireless links are very small, then propagation delay is assumed to be negligible [20].

\subsection{Estimation of Available Bandwidth}

We use the mechanism suggested by [21] for the estimation of available bandwidth by using the idle period of the wireless channel. The idle period of a wireless channel, being an indication of traffic travelling along the mobile nodes as well as their neighbourhoods, is a key factor for bandwidth estimation. A mobile node can transmit data frames successfully during this idle period. The formula for calculation of available bandwidth can be described through:

$$
B W_{A v}=B W_{\text {Max }} *\left(\frac{I d l e_{t}}{\text { Int }_{t}}\right)
$$


where $B W_{M a x}$ is the maximum bandwidth of the link and Idle $_{t}$ is the wireless channel's idle duration over a time interval Int $t_{t}$. The virtual carrier sensing method provided by the IEEE 802.11 MAC standard can determine the state of the wireless channel and can be utilised to observe the channel state transitions. In a unit time interval, if $B u s y_{t}$ denotes the period during which the channel remains busy, then the period during which the channel remains idle, Idle $e_{t}$ can be defined as:

$$
\text { Idle }_{t}=\text { Int }_{t}-\text { Busy }_{t}
$$

Substituting the value of $I d l e_{t}$ into Equation (7), $B W_{A v}$ can be calculated [21].

\subsection{Estimation of Load}

We use the mechanism based on contention window $(\mathrm{CW})$ size and queue length proposed in [22] for estimation of load in a node. The IEEE 802.11 MAC provides contention based Distributed Coordination Function (DCF) as the basic method of channel access. The collision avoidance mechanism of DCF uses the 'binary exponential back-off' algorithm, where contenting nodes are randomly given a 'back-off interval' between zero and CW (contention window). Nodes wait for the given number of time slots before trying to access the channel again. The size of $\mathrm{CW}$ is doubled when there is a collision, till reaching a maximum value $C W_{\text {Max }}$. Hence $C W$ gives a good indication of contention of the channel and can be used to estimate the load around a node [22]. Traffic over a long period is estimated to alleviate the effect of traffic bursts. The computation of a node's $C W$ is carried out every $t$ seconds. $C W$ is calculated by applying the 'exponential weighted moving average' method using the old contention window $C W_{\text {old }}$ and the current contention window $C W_{\text {current }}$ as given by:

$$
C W=x \times C W_{\text {old }}+(1-x) \times C W_{\text {current }}
$$

where $x$ is a smoothing constant. To grant higher priority to $C W_{\text {current }}, x$ is set to 0.3 to provide a better indication of the current status of a node [22].

The packet count in the interface queue of a node can be considered as an indicator of the traffic load of the node. The larger the number of packets in the queue, the greater will be the traffic load on that node. Thus the average queue size can be used as an indicator for traffic load at a node over a long time period. The following formula is used to calculate the average queue size of a node which is updated every $t$ seconds (see [22]):

$$
Q_{\text {len }}=y \times \text { qlen }_{\text {old }}+(1-y) \times \text { qlen }_{\text {current }}
$$

where Qlen is the average length of queue, qlen $_{\text {current }}$ is the current queue length and $y$ is another smoothing constant which is set to 0.3 . The parameters $x$ and $y$ can be any number selected between 0 and 1. Using the values of $C W$ and Qlen, the following equation can be used to calculate load at node $i$ :

$$
\operatorname{Load}_{i}=z \times\left(\frac{C W}{M a x C W}\right)+(1-z) \times\left(\frac{Q_{\text {len }}}{M a x Q_{l e n}}\right)
$$

The constant $z$ is used to control the effects of the two factors of $C W$ and Qlen. In this work $z$ is set to 0.5 , which ensures that equal priority is given to both factors. As can be seen in (11), smaller the values of $Q_{l e n}$ and $C W$ indicate a lower load and vice versa [22].

\subsection{Estimation of Residual Battery Energy}

NS-2 [23] provides an energy model, defined as a node attribute, by the class EnergyModel, which is simple and represents the energy level in a mobile node [24, 25]. It defines transmit, receive, idle and sleep as four radio states which are represented respectively by parameters txPower, rxPower, idlePower, and sleepPower. These parameters contain the quantity of energy spent per unit time for each state. The energy consumed for every transmission and reception of packets by the node is decreased from the initialEnergy, which represents the energy of a node at the beginning of the simulation. No more packets can be transmitted or received by the node when its energy level subsequently becomes zero. The energy consumed in transmitting state $E_{t x}$ during a time txTime is: $E_{t x}=$ txPower $\times$ txTime. In the same way, the energy consumed in receiving state $E_{r x}$ during a time rxTime is: $E_{r x}=$ rxPower $\times$ rcvTime [24]. The residual energy of each node $E_{r e s}$ can then be estimated as:

$$
E_{r e s}=E_{i n i}-\left(E_{t x}+E_{r x}\right)
$$

where $E_{i n i}$ is the initialEnergy which is set to $14.1 \mathrm{~J}$ in the simulations. This value is found to be sufficient to keep the nodes alive till the end of simulation. idlePower and sleepPower are not included in the model, being very small compared to txPower and rxPower.

\section{FUZZY BASED LOAD DISTRIBUTION}

The traffic distribution decision is performed using fuzzy logic techniques. Figure 1 shows the steps involved in the fuzzy based load distribution system in a mobile node. The four discrete input variables to be fuzzified are Delay, Available BW, Load and Residual Energy. The membership functions 'LOW' and 'HIGH' are used to describe each of these input variables. The output is the load distribution possibility (LDP) at a node for the current conditions of the input variables. For the output, we take three values: 'LOW', 'MEDIUM' and 'HIGH'. Figures 2 and 3 show the membership functions for input and output variables respectively. Due to their computational efficiency and simple formulas, triangular functions are used as the membership functions since these are widely used in real-time applications. Table 1 outlines the fuzzy inference system that connects the inputs (RE, L, D and Av. BW) with the output LDP at a node. Each row in the table represents a fuzzy rule similar to the human thinking process.

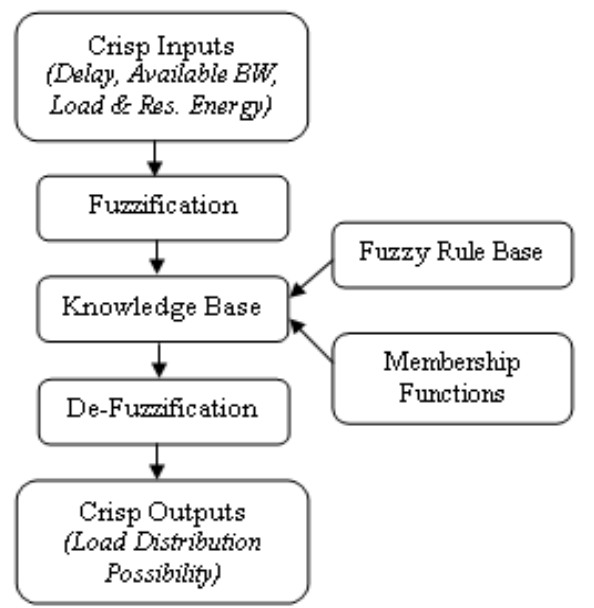

Figure 1: Fuzzy Based Load Distribution System 

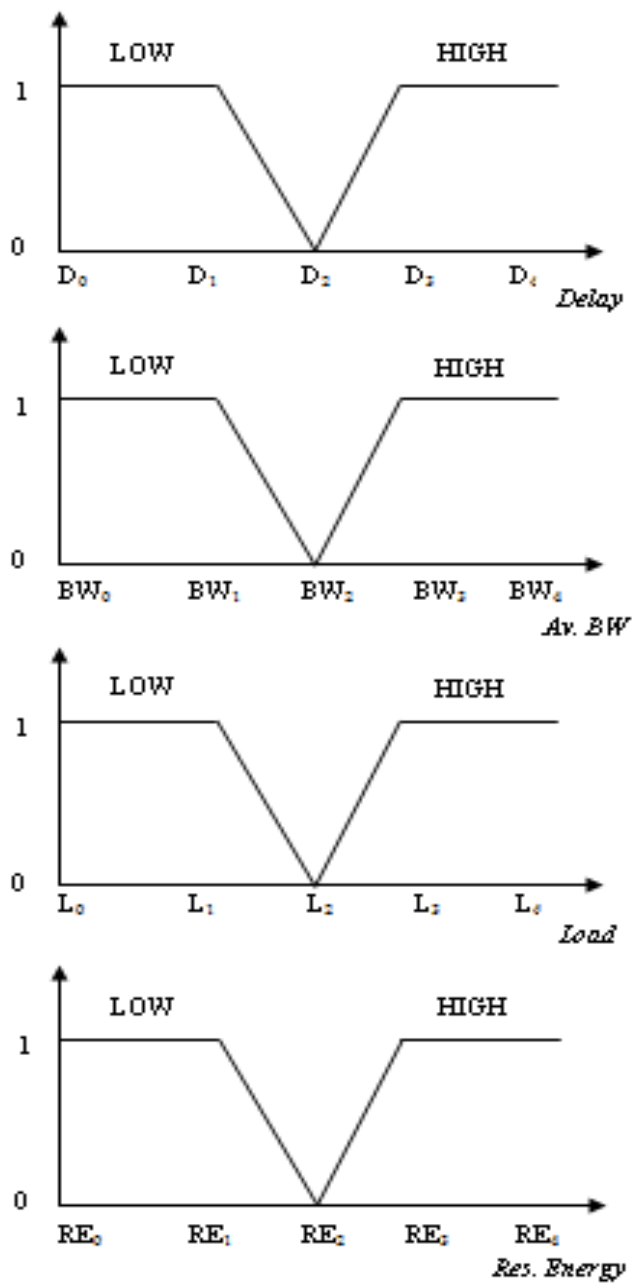

Figure 2: Input Membership Functions

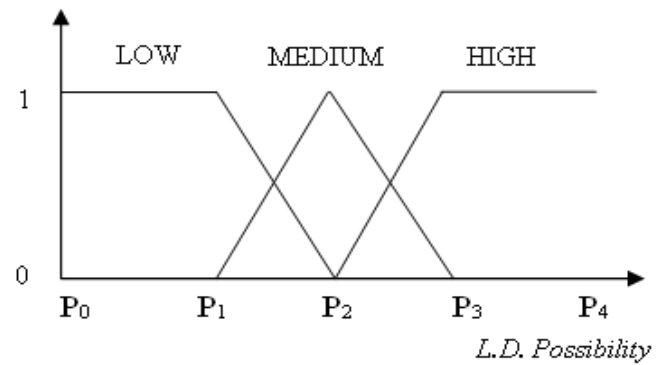

Figure 3: Output Membership Function

Table 1: Fuzzy Inference Rules

\begin{tabular}{|l|l|l|l|l|}
\hline $\begin{array}{l}\text { Residual } \\
\text { Energy } \\
(\mathrm{RE})\end{array}$ & $\begin{array}{l}\text { Load } \\
(\mathrm{L})\end{array}$ & $\begin{array}{l}\text { Delay } \\
\text { (D) }\end{array}$ & $\begin{array}{l}\text { Available } \\
\text { BW } \\
\text { (Av. BW) }\end{array}$ & $\begin{array}{l}\text { L.D. } \\
\text { Possibility } \\
\text { (LDP) }\end{array}$ \\
\hline High & Low & Low & High & High \\
\hline High & Low & Low & Low & Medium \\
\hline Low & Low & Low & High & Medium \\
\hline High & High & Low & High & Medium \\
\hline High & Low & High & High & Medium \\
\hline High & High & High & High & Low \\
\hline
\end{tabular}

\begin{tabular}{|l|l|l|l|l|}
\hline $\begin{array}{l}\text { Residual } \\
\text { Energy } \\
\text { (RE) }\end{array}$ & $\begin{array}{l}\text { Load } \\
\text { (L) }\end{array}$ & $\begin{array}{l}\text { Delay } \\
\text { (D) }\end{array}$ & $\begin{array}{l}\text { Available } \\
\text { BW } \\
\text { (Av. BW) }\end{array}$ & $\begin{array}{l}\text { L.D. } \\
\text { Possibility } \\
\text { (LDP) }\end{array}$ \\
\hline Low & High & High & Low & Low \\
\hline Low & High & Low & Low & Low \\
\hline Low & Low & High & Low & Low \\
\hline Low & Low & Low & Low & Low \\
\hline
\end{tabular}

The fuzzy inference system employed in this work has ten rules with the combinations as presented in the Table 1. For example, the first rule in Table 1 stands for 'If RE is high, Delay is low, Load is low and BW is high, then LDP is high'. The ideal condition for distribution possibility will be high residual energy, low delay, low load and high available bandwidth. Since this rule satisfies the ideal condition, LDP is high, and load is distributed whenever the fuzzy based system provides 'HIGH' or 'MEDIUM' load distribution possibilities.

\subsection{Fail-Safe Multipath Discovery}

The multipath route discovery presented in this work is based on SMORT [7], which is an AODV based scalable multipath routing protocol. It achieves scalability by using alternate paths which reduce routing overhead due to additional route creations and route error messages which occur in single path on-demand protocols. Fail-safe multiple paths are computed for nodes in the primary path which are used for re-directing traffic when nodes on the main path move away or fail. SMORT uses special route-request and route-reply packet structures for computing these multiple paths from sender to receiver. The route discovery phase is started by a node through network wide flooding of a route-request, when a route is required to some destination node. All the intermediate nodes that have a suitable path to the receiver send a route-reply packet back to the sender upon receiving the request. Otherwise, they rebroadcast (the first copy of) the route-request. Nodes accept multiple copies of the route-request to facilitate the construction of multiple fail-safe routes. A request-rcvd table stores all route-request copies received at a node. A route-reply packet is sent back to the source by the destination in the route reply phase when it subsequently receives the request. [7].

When the first route is created, the source eventually begins data transmission to the destination. Though multiple routereply packets may arrive at intermediate nodes, because multiple replies are sent by the destination to multiple copies of the route requests, they relay only the first route-reply. To relay the route-reply, the neighbours use the nodes that they received the route-request previously. Nodes drop extra replies after updating their routing tables with secondary routes contained in them. If all the routes between source and destination nodes fail during a data transmission session, a route maintenance phase is invoked to re-establish the connection. Expired routes are removed from the nodes' routing tables. SMORT avoids routing loops during the route-reply phase by allowing routereply packets to carry full path information to the destination. These multiple paths avoid the need for fresh route creation when a route/node failure occurs in the primary path. In [7] the authors have demonstrated that the primary path and fail-safe secondary paths are loop-free.

\section{SIMULATION ENVIRONMENT, RESULTS AND ANALYSIS}

NS-2 [23] has been used to simulate the new protocol. The channel capacity of mobile nodes was set to $2 \mathrm{Mbps}$. The 
simulation settings and parameters used in the model are summarised in Table 2. The proposed protocol is compared with AOMDV [26] with the same settings and parameters mentioned above. AOMDV is the multipath version of the popular AODV protocol that is designed primarily for highly dynamic ad hoc networks where frequent link failures and route breaks occur. AOMDV provides multiple redundant paths from source to destination. The performance of FLEAMR has been investigated for constant-bit-rate (CBR) traffic using packet end-to-end delay, packet delivery ratio (PDR), packet drop and average energy consumption as measured parameters. The numbers of nodes simulated are 30, 50, 70, 90 and 110, with speeds of $10 \mathrm{~m} / \mathrm{s}$. The simulations were repeated for different data rates from $250 \mathrm{Kbps}$ to $450 \mathrm{Kbps}$ keeping the number of nodes at 110 and speed at $10 \mathrm{~m} / \mathrm{s}$. The random waypoint mobility model has been used with $5 \mathrm{sec}$ pause time in a field of dimension $1250 \times 1250 \mathrm{~m} .5$ active traffic sessions were maintained in all scenarios. Figures 4 to 8 show the results for varying numbers of nodes while Figures 9 to 13 show the results for varying data rates.

Table 2: Simulation Settings

\begin{tabular}{|l|l|}
\hline Number of Nodes & $30,50,70,90$ and 110 \\
\hline Area & 1250 X $1250 \mathrm{~m}$ \\
\hline MAC Protocol & IEEE $802.11 \mathrm{DCF}$ \\
\hline Radio Range & $250 \mathrm{~m}$ \\
\hline Simulation Time & $90 \mathrm{~s}$ \\
\hline Traffic Type & CBR \\
\hline Packet Size & $512 \mathrm{~B}$ \\
\hline Speed & $10 \mathrm{~m} / \mathrm{s}$ \\
\hline Pause Time & $5 \mathrm{sec}$ \\
\hline Rate & $250 \mathrm{~Kb} / \mathrm{s}$ to $450 \mathrm{~Kb} / \mathrm{s}$ \\
\hline Mobility Model & Random Way Point \\
\hline Tx Power & $0.660 \mathrm{~W}$ \\
\hline Rx Power & $0.395 \mathrm{~W}$ \\
\hline Initial Energy & $14.1 \mathrm{~J}$ \\
\hline
\end{tabular}

Figure 4 reveals that for CBR data, the packet end-to-end delay is considerably less for FLEAMR compared to AOMDV, particularly for higher numbers of nodes. The reason is that for higher numbers of nodes, there will possibly be more fail-safe multiple routes to the destination. A maximum saving of about $100 \mathrm{~ms}$ is seen in packet delay between FLEAMR and AOMDV for 90 nodes. In Figures 5, 6 and 7, FLEAMR also show considerable improvement in PDR, throughput and packet drop when compared to AOMDV. A maximum of $35 \%$ improvement in PDR and 300kbps improvement in throughput and a reduction in packet drop by 5000 is observed between FLEAMR and AOMDV. Figure 8 reveals that considerable saving is achieved in average energy consumption with a maximum of around 5 Joules being observed for 30 nodes.

Figures 9 to 13 show that FLEAMR also performs better under varying data rates. Figure 9 shows an improvement of around 90ms in delay compared to AOMDV which is steady at almost all data rates considered. Figures 10,11 and 12 respectively show a maximum $35 \%$ increase in PDR, an increase of 400 kbps in throughput and a reduction in packet drop by around
8000 while Figure 13 shows a steady saving in energy consumption by a factor of around 2 Joules.

The reason for the performance improvements may be attributed to the fact that the protocol employs a cross layer approach to capture the forwarding delay, load, available bandwidth and residual energy at various locations in the network and selects multiple paths which are rich in resources and less congested. Even if a node is not congested, it cannot be used as a router if its battery energy is on the verge of depletion and this scenario is recognised by the protocol. The results for higher node density reveal that the protocol scales well. Similarly, the performance of the protocol is robust and steady at higher data rates for CBR traffic.

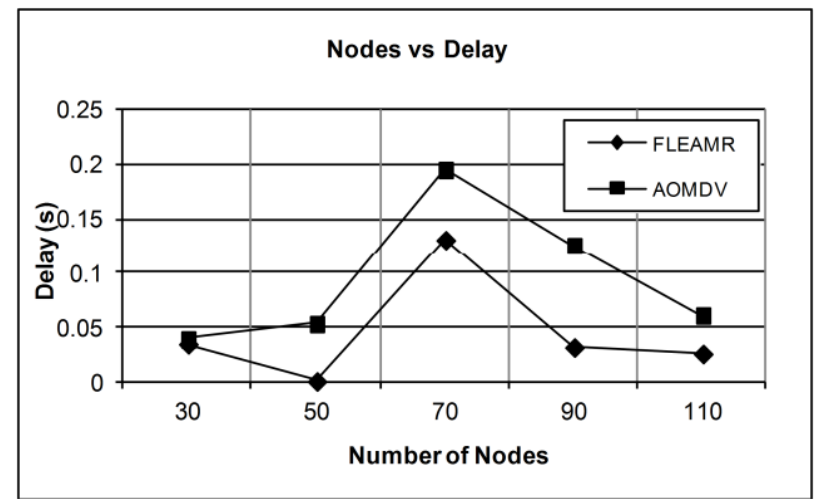

Figure 4: Number of Nodes vs. End-to-End Delay

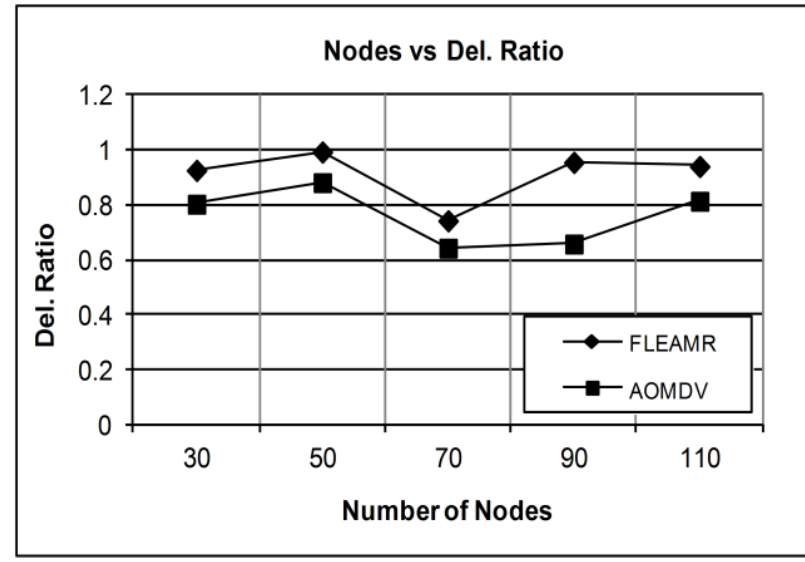

Figure 5: Number of Nodes vs. Delivery Ratio

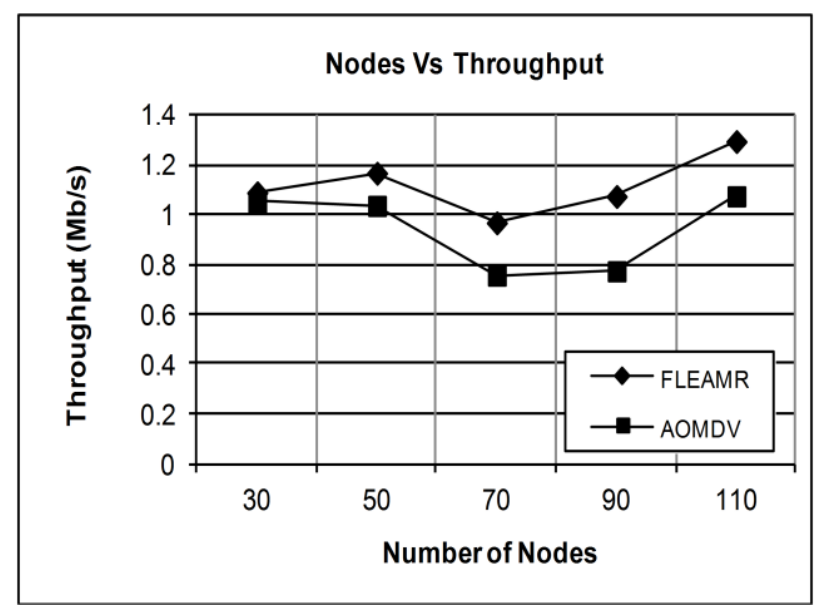

Figure 6: Number of Nodes vs. Throughput 


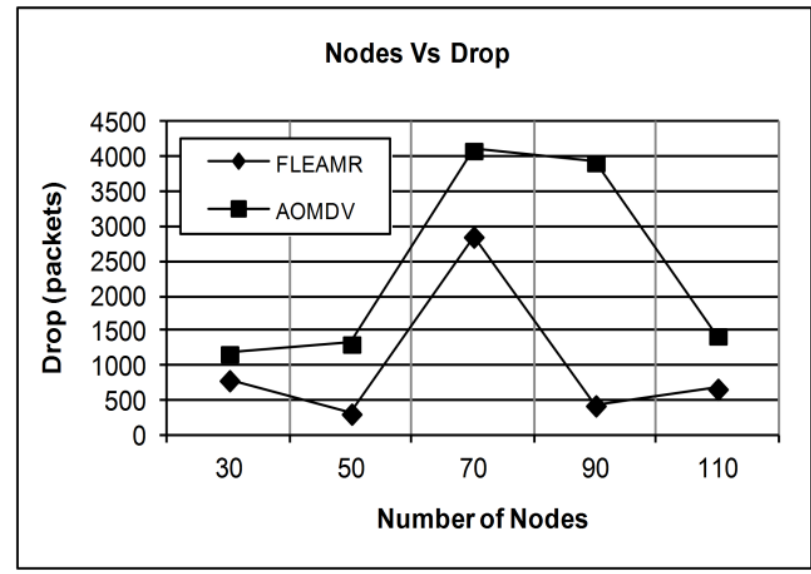

Figure 7: Number of Nodes vs. Packet Drop

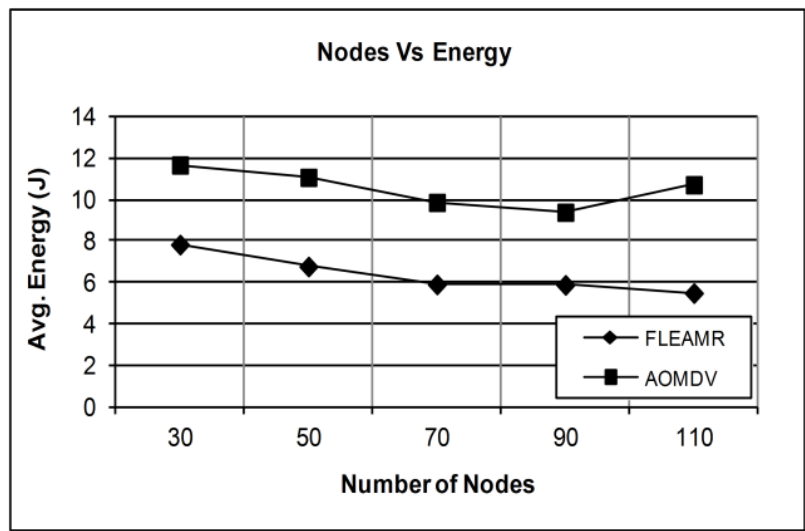

Figure 8: Number of Nodes vs. Avg. Energy Consumption

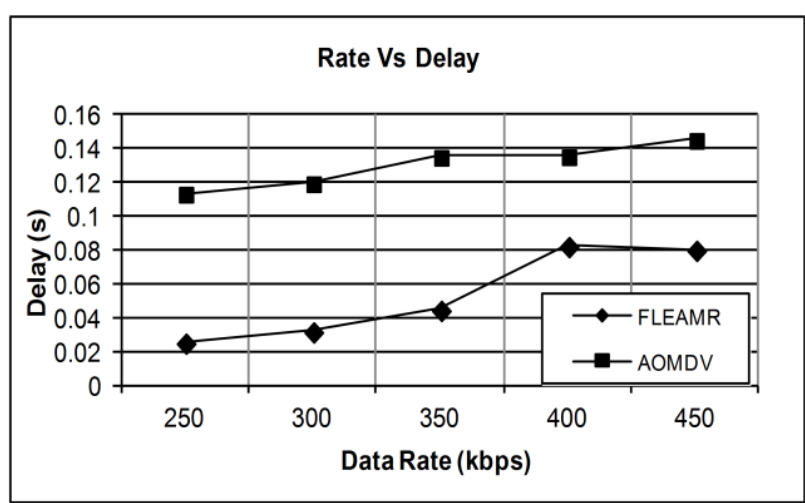

Figure 9: Data Rate vs. End-to-End Delay

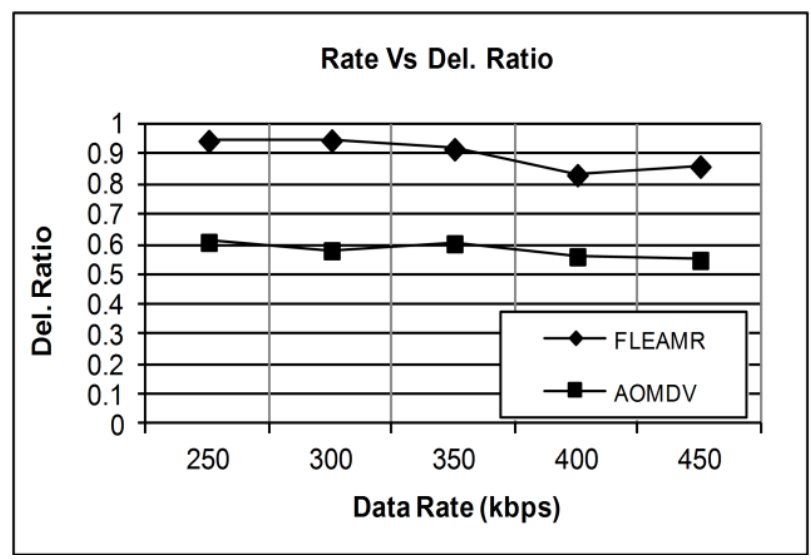

Figure 10: Data Rate vs. Delivery Ratio

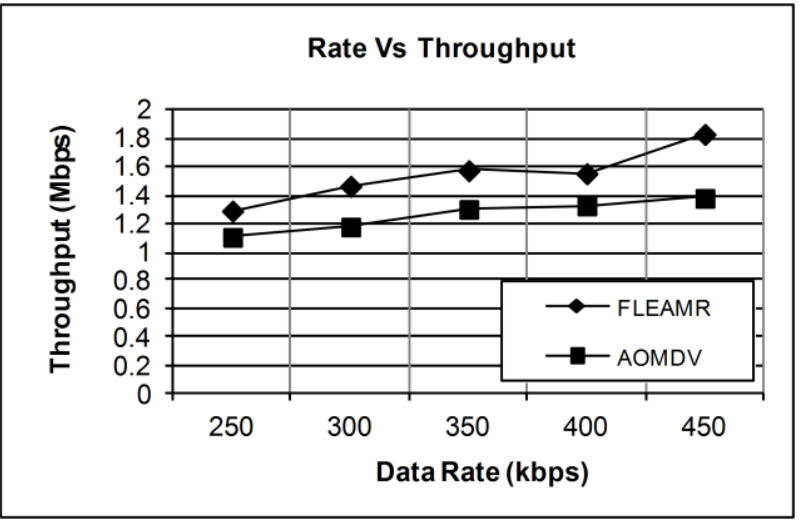

Figure 11: Data Rate vs. Throughput

Thus FLEAMR accurately captures and manages congestion throughout the network. It is expected that by avoiding overburdening of nodes which are used for routing of packets, the protocol will improve energy balancing of nodes which may increase network life time considerably.

Figure 12: Data Rate vs. Packet Drop

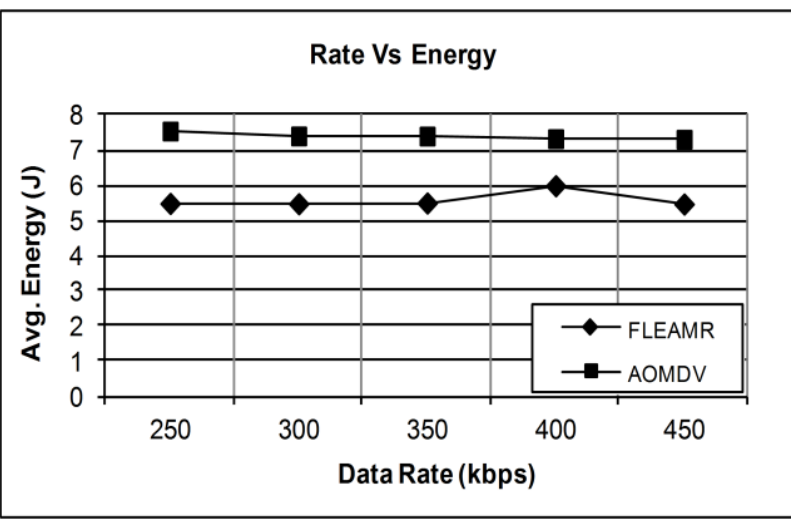

Figure 13: Data Rate vs. Average Energy Consumption

\section{CONCLUSION}

A fuzzy based load and energy aware multipath routing protocol has been proposed for application within MANETs. The forwarding delay, available bandwidth, load and residual battery energy of a node are given as inputs to a fuzzy inference engine to determine the traffic distribution possibility from that node based on the given fuzzy rules. Based on the output from the fuzzy system, traffic is distributed over failsafe multiple routes to the destination to reduce the load at a congested node. Thus the protocol captures delay, load, available bandwidth and energy consumption at various locations of the network and effectively manages them resulting in improved network performance. Traffic is distributed over fail-safe multipath routes to the destination whenever the fuzzy based system gives 'HIGH' or 'MEDIUM' load distribution possibilities as output. Simulation results show that the protocol improves packet delivery ratio and throughput with reduced delay and average energy consumption when compared to the popular AOMDV protocol. Since the protocol is multipath, the routing overhead may be slightly higher. Further, the protocol has been tested only for CBR traffic. Load balancing of a variable bit-rate (VBR) video stream in the network is critical to ensure the quality of the received data. Hence the future work aims to evaluate both routing overhead influences and the effect of real-time VBR traffic using the proposed protocol. 


\section{REFERENCES}

[1] S. Mueller, R. P. Tsang and D. Ghosal, "Multipath Routing in Mobile Ad Hoc Networks: Issues and Challenges", Invited paper in lecture notes in Computer Science, Edited by Maria Carla Calzarossa and Erol Gelenbe, 2004

[2] S. Santhi and G. S. Sudha, "Enhanced AOMDV for Energy Conservation in Hybrid Ad hoc Networks", International Journal of Information and Communication Technology, Vol. 6, No. 2, pp. 122-141, April 2014.

[3] R. Kumar, A. K Sarje and M. Misra, “An AODV based QoS Routing Protocol for Delay Sensitive Applications in Mobile Ad Hoc Networks", Journal of Digital Information Management, Vol 8 No. 5, October 2010.

[4] J. Y. Choi and Y. B. Ko, "Multi-path Routing with Loadaware Metric for Tactical Ad Hoc Networks", International Conference on Information and Communication Technology Convergence (ICTC), Jeju, 17-19 Nov, 2010.

[5] O. Souihli, M. Frikha and M. B Hamouda, "Loadbalancing in MANET shortest-path routing protocols", in proc. of Journal on Ad Hoc Networks, vol. 7, no. 2, p. 431- 442, March 2009.

[6] S. P. Terdal, V. D. Mytri, A. Damodaram and B. Salimath, "Multipath Load Balancing: An Entropy based Clustering Solution for Mobile Ad hoc Networks", International Journal on Recent Trends in Engineering \& Technology, Vol. 05, No. 01, Mar 2011.

[7] L. R. Reddy and S.V. Raghavan, "SMORT: Scalable multipath on-demand routing for mobile ad hoc networks", in proc. of Journal on Ad Hoc Networks, vol. 5, no. 2, p. 162- 188, March 2007.

[8] M. Ali, B.G. Stewart, A. Shahrabi and A. Vallavaraj, "QoS Aware Multipath Threshold Routing for Mobile Ad hoc Networks", International Journal of Applied Information Systems (IJAIS), Vol. 7, No. 1, p. 8-15, April 2014.

[9] P. Nayak, R. Agarwal and S. Verma, "An Overview of Energy Efficient Routing Protocols in Mobile Ad Hoc Networks", International Journal of Research and Reviews in Ad hoc Networks (IJRRAN), Vol. 2, No. 1, p. 93-96, March 2012.

[10] A. Kush, D. Sharma and S. Taneja, "A Secure and Power Efficient Routing Scheme for Ad Hoc Networks", International Journal of Computer Applications, Vol. 21, No. 6, p. 20-15, May 2011.

[11] V. Rishiwal, M. Yadav, S. Verma and K. Bajapai, "Power Efficient Routing in Ad Hoc Wireless Networks", in proc. of Journal of Computer Science \& Technology, Vol. 9, No. 2, p. 101-109, October 2009.

[12] P. Nayak, R. Agrawal and S. Verma, "Energy Aware Routing Scheme for Mobile Ad hoc Network Using Variable Range Transmission", in Intl. Journal of Ad hoc, Sensor \& Ubiquitous Computing (IJASUC) Vol.3, No.4, August 2012.

[13] P. Srinivasan and P. Kamalakkannan, "RSEA-AODV: Route Stability and Energy Aware Routing for Mobile Ad Hoc Networks", International Journal of Computer Communication, Vol. 8, No. 6, p. 891-900, Dec 2013.
[14] P. Pham and S. Perreau, "Performance Analysis Of Reactive Shortest Path And Multi-Path Routing Mechanisms With Load Balance", IEEE Conference on Computer Communications (INFOCOM 2003), March 2003.

[15] S. Ghosh, Q. Razouqi, H.J. Schmacher and A. Celmis, "A Survey of Recent Advances in Fuzzy Logic in Telecommunication Networks and New Challenges", IEEE Transactions on Fuzzy Systems, Vol. 6.No.3, p. 443447, August 1998.

[16] E. Natsheh, S. Khatun and A.B. Jantan, "Adaptive Fuzzy Route Lifetime for Wireless Ad hoc Networks", The International Arab Journal of Information Technology, Vol. 3, No. 4, p. 283-290, October 2006.

[17] A.A. Ali and T.O. Fahad, "Fuzzy Energy based Routing Protocol for MANET", International Journal of Computer Applications, Volume 46, No.1, p. 38-40, May 2012.

[18] R. de Oliveira and T. Braun, "A Delay-based Approach Using Fuzzy Logic to Improve TCP Error Detection in Ad Hoc Networks", In proc. of IEEE Wireless Communications and Networking Conference (WCNC 2004), Vol. 3, p. 1666 - 1671, 2004.

[19] S. Misra, S. Roy, M.S. Obaidat, D. Mohanta, “A Fuzzy Logic-Based Energy Efficient Packet Loss Preventive Routing Protocol", In proc. of International Symposium on Performance Evaluation of Computer \& Telecommunication Systems - SPECTS 2009, Istanbul, Turkey, Vol. 41, pp. 185 - 192, 13-16 July 2009.

[20] S. T Sheu and J. Chen, "A Novel Delay Oriented Shortest Path Routing Protocol for Mobile Ad Hoc Networks" ICC 2001 The IEEE International Conference on Communications, Vol 9, pp 1930-34, June 2001.

[21] F. Qin and Y. Liu, "Multipath Routing for Mobile Ad Hoc Network", Proc. of the 2009 International Symposium on Information Processing (ISIP'09) Huangshan, P. R. China, pp. 237-240, August 21-23, 2009.

[22] X. Gao, X. Zhang, D. Shi, F. Zou and W. Zhu, "Contention and Queue-aware Routing Protocol for Mobile Adhoc Networks", International Conference on Wireless Communications, Networking and Mobile Computing (WiCom 2007), Shanghai, 21-25 Sept. 2007.

[23] Network Simulator, http://www.isi.edu/nsnam/ns, Accessed on $1^{\text {st }}$ June 2014.

[24] F. Faoudi, S. Yahiaoui and Y. Belhoul, "NS-2 Based Framework for Topology Control in Wireless Ad-hoc Networks", Journal of Communications in Information Science and Management Engineering, Vol. 2, Issue 4, P.1-7, 2012.

[25] K. Fall and K. Varadhan, "The NS Manual", The VINT project, UC Berkeley, LBL, USC/ISI, and Xerox PARC, available at http://www.isi.edu/nsnam/ns/doc/ 2011, Accessed on $1^{\text {st }}$ June 2014.

[26] M. K Marina and S. R Das, "On Demand Multipath Distance Vector Routing in Ad hoc Networks", in Proc. of the Ninth International Conference on Network Protocols, p. 14-23, 2001. 